Special issue in honor of Prof. George C. Papageorgiou

\title{
Sensing and classification of rice (Oryza sativa L.) drought stress levels based on chlorophyll fluorescence
}

\author{
Q. XIA*, L.J. FU*, H. TANG*, L. SONG ${ }^{* *}$, J.L. TAN***, and Y. GUO*,**,+ \\ Key Laboratory of Advanced Process Control for Light Industry, Ministry of Education, School of IoT, Jiangnan \\ University, 214122 Wuxi, China* \\ Joint International Research Laboratory of Agriculture and Agri-Product Safety, Yangzhou University, 225009 \\ Yangzhou, China ${ }^{* *}$ \\ Department of Bioengineering, University of Missouri, Columbia, MO 65211, USA $A^{* * *}$
}

\begin{abstract}
Sensing and classification of drought stress levels are very important to agricultural production. In this work, rice drought stress levels were classified based on the commonly used chlorophyll $a$ fluorescence $(\mathrm{ChlF})$ parameter $\left(\mathrm{F}_{\mathrm{v}} / \mathrm{F}_{\mathrm{m}}\right)$, feature data (induction features), and the whole OJIP induction (induction curve) by using a Support Vector Machine (SVM). The classification accuracies were compared with those obtained by the K-Nearest Neighbors (KNN) and the Ensemble model (Ensemble) correspondingly. The results show that the SVM can be used to classify drought stress levels of rice more accurately compared to the KNN and the Ensemble and the classification accuracy $(86.7 \%)$ for the induction curve as input is higher than the accuracy $(43.9 \%)$ with $F_{v} / F_{m}$ as input and the accuracy $(72.7 \%)$ with induction features as input. The results imply that the induction curve carries important information on plant physiology. This work provides a method of determining rice drought stress levels based on ChlF.
\end{abstract}

Keywords: chlorophyll $a$ fluorescence; drought stress; Ensemble model; K-Nearest Neighbors; Support vector machine.

\section{Introduction}

With global climate and environmental changes, the frequency and duration of irregular precipitation and continuous drought stress caused by extreme weather are increasing, and the degree of crop damage and loss is also rising (Webber et al. 2018, Xu et al. 2020). In addition, pollution further exacerbates the water resource crisis.
As a major crop, rice demands a high level of soil moisture (Carrijo et al. 2018). Water is tightly connected with crop photosynthetic capacity, which can seriously impact the yield and quality of rice (Rosegrant and Cline 2003, Xu et al. 2020). With the shortage of water, drought stress has become an important factor in reducing rice yield (Iturbe-Ormaetxe et al. 1998, Yang et al. 2019, Melandri et al. 2020). Thus, to improve rice production and reduce

\section{Highlights}

- An SVM-based method was developed to classify rice drought stress levels

- All the data on the chlorophyll fluorescence induction OJIP were used as inputs

- Results were more accurate than analysis with only using some feature parameters
Received 21 July 2021

Accepted 25 January 2022

Published online 28 February 2022

${ }^{+}$Corresponding author

e-mail: guoy@jiangnan.edu

Abbreviations: ChlF - chlorophyll a fluorescence; D0 - no drought stress treatment; D1 - drought stress treatment for 1 h; D4 - drought stress treatment for $4 \mathrm{~h}$; KNN - K-Nearest Neighbors; OJIP - chlorophyll $a$ fluorescence induction kinetics curve; SVM - Support Vector Machine.

Acknowledgments: This project is partially supported by the National Natural Science Foundation of China (No. 31771680, 51961125102), and the 111 Project (B12018).

Conflict of interest: The authors declare that they have no conflict of interest. 
irrigation costs, it is of great significance to study rice drought stress measures (Sayed 2003).

Photosynthesis is an important physiological process, which is closely related to plant water status, and it is very sensitive to water shortage. Photosynthetic rate decreases under drought stress (Miyashita et al. 2005). When leaf water content drops to a certain extent, plant photosynthesis will be inhibited or even completely stopped. Drought events are usually characterized by drought indices (Marcos-Garcia et al. 2017, Mukherjee et al. 2018). In agriculture, drought monitoring methods can be divided into three categories: meteorological monitoring methods (Jiao et al. 2019), remote sensing methods (Kumar et al. 2014, West et al. 2019), and integrated remote sensing monitoring methods (Haboudane et al. 2004, Sánchez et al. 2018). However, these methods do not directly relate to photosynthetic efficiency. There is still a lack of universal, noninvasive, convenient, and affordable plant-physiologybased measures of drought stress that can reflect the influence of drought on photosynthesis.

ChlF emission from photosystem II (PSII) couples with light energy absorption, transmission, dissipation, and distribution, and it plays an important role in determining photosynthesis (Lazár 2006, Maghsoudi et al. 2015, Urban et al. 2017). It can sense the effects of the external environment on the photosynthesis and physiology of plants, even if the changes in the external environment are very subtle. It is widely used in photosynthesis analysis, plant stress physiology research, oceanography, and remote sensing (Feng et al. 2018, Xia et al. 2018, Fu et al. 2019, Wang et al. 2019), and even genetic breeding under drought environments (Woo et al. 2008, Faraloni et al. 2011, Guo and Tan 2015, Zendonadi et al. 2021). ChlF measurement does not damage plant cells or cause harm to organisms. Thus, it may be used to measure the effect of drought on rice (Maghsoudi et al. 2015).

In the literature, researchers studied the influence of environmental factors on ChlF to indirectly study the influence of the environment on plant photosynthesis. The minimum dark ChlF $\left(\mathrm{F}_{\mathrm{o}}\right)$, the maximum dark ChlF $\left(\mathrm{F}_{\mathrm{m}}\right)$, and the parameters calculated according to some feature data on the ChlF induction are often used for analysis (Banks 2018, Meravi and Prajapati 2020). At the same time, most of the data on the measured ChlF induction curve is not used for the analysis of plants; however, it may contain useful information and enhance the signal-to-noise ratio.

Support Vector Machine (SVM) is a very effective classification tool and has been widely used in agricultural plant recognition. SVM algorithm is a supervised learning model defined by separating hyperplanes based on the statistical learning theory. It is a method in the field of artificial intelligence and data mining. The generalization performance of SVM focuses on the principle of structural risk minimization (SRM), rather than minimizing the error in the training data, which thus avoids common overfitting problems in most Artificial Neural Network (ANN) models. Therefore, in an SVM model, even if the dimensionality of the training samples is very high, SVM can obtain high generalization ability. A large number of applications also show that SVM has good classification capabilities in processing a small number of samples, nonlinear, and high-dimensional pattern recognition.

SVM was widely applied in agriculture, for example, material identification (Karimi et al. 2006, Guerrero et al. 2012, Kour and Arora 2019, Qiao et al. 2019), adulteration percentage classification (Timsorn et al. 2017), plant substance content prediction (Ignat et al. 2013, Wang et al. 2018), and disease classification (Alam and Kwon 2017). Although SVM has been widely used in agricultural plants, it has not been used in plant drought stress recognition based on ChlF.

Drought stress is one of the main environmental factors that restrict rice growth. The damage of drought stress to rice growth is multifaceted and the response mechanism of rice to drought stress is also extremely complex, which can lead to rice yield reduction and even death. The research on rice drought stress response has reached the molecular level, involving cloning, positioning, and functional analysis of drought resistance genes. However, there is a lack of a universal, noninvasive, convenient, and economic method for determining drought stress levels based on plant physiology to reflect the impact of drought stress on photosynthesis. Therefore, in this work, SVM was used to classify rice drought stress levels with the entire OJIP induction curve or computed induction feature(s) as input variables. In addition, the K-Nearest Neighbors (KNN) and the Ensemble methods were used for comparison.

\section{Materials and methods}

Experimental samples: Rice plants (Oryza sativa L.) were uprooted from a production field at $6: 30 \mathrm{~h}$ in July 2019 after growing for about $40 \mathrm{~d}$. To reduce initial differences in moisture contents, the roots of rice were immersed in water for two hours. Then the roots were put into a $20 \%$ polyethylene glycol (PEG) solution for different durations (D0 - no drought stress treatment; D1 - drought stress treatment for $1 \mathrm{~h}$; D4 - drought stress treatment for $4 \mathrm{~h}$ ) to achieve different levels of drought stress. PEG-induced drought is commonly used in plant drought stress research (Wang et al. 2005, Cai et al. 2015, Faseela et al. 2020). We used 883 rice plants.

Fluorescence measurement: $\mathrm{ChlF}$ of the treated plant samples was measured with a FluorPen hand-held fluorometer (Model FF 110, Photon Systems Instruments, Czech Republic) set to its OJIP protocol.

Data analysis: The measured $\mathrm{ChlF}$ induction responses were used as inputs and the drought level (D0, D1, and D4) as the classifier or output. The induction responses were used in three ways: (1) a ChlF parameter: maximum photochemical quantum yield of PSII in the dark $\left(\mathrm{F}_{\mathrm{v}} / \mathrm{F}_{\mathrm{m}}\right)$; (2) induction features: computed features of ChlF induction $\left(\mathrm{F}_{\mathrm{o}}, \mathrm{F}_{\mathrm{j}}, \mathrm{F}_{\mathrm{i}}, \mathrm{F}_{\mathrm{m}}, \mathrm{F}_{\mathrm{v}}, \mathrm{V}_{\mathrm{j}}, \mathrm{V}_{\mathrm{i}}, \mathrm{F}_{\mathrm{m}} / \mathrm{F}_{\mathrm{o}}, \mathrm{F}_{\mathrm{v}} / \mathrm{F}_{\mathrm{o}}, \mathrm{F}_{\mathrm{v}} / \mathrm{F}_{\mathrm{m}}, \mathrm{M}_{0}\right.$, Area, Fix Area, $\mathrm{S}_{\mathrm{m}}, \mathrm{S}_{\mathrm{s}}, \varphi \mathrm{P}_{0}, \psi_{0}, \varphi \mathrm{E}_{0}, \varphi \mathrm{D}_{0}, \varphi \mathrm{Pav}, \mathrm{PI}_{\mathrm{ABS}}$, $\mathrm{ABS} / \mathrm{RC}, \mathrm{TR}_{0} / \mathrm{RC}, \mathrm{ET}_{0} / \mathrm{RC}$, and $\mathrm{DI}_{0} / \mathrm{RC}$ ) (see Appendix for a more detailed explanation about ChlF parameters); 
(3) induction curve: all the data of the ChlF induction curve. Before analysis or feature computation, the ChlF induction data were normalized by using the Min-Max method.

Statistical analysis was performed on the induction features with the statistical software package SPSS (SPSS Inc., version 21.0, Chicago, USA). Statistical differences between different levels of drought stress were determined by using the least significant difference (LSD) method at the 0.05 probability level.

SVM, KNN, and the Ensemble toolbox in MATLAB (The MathWorks, Natick, MA, USA) were used to classify the plant samples into the three drought levels. The parameters or settings for each method were determined experimentally for the best classification accuracy. The cubic kernel was used as the SVM kernel function, the kernel scale was set to 'automatic', 'boxconstraint' level to 1 , and the multiclass method to 'one- $v s$-one'. The distance metric of KNN was set as 'cosine', the number of neighbors was set as 10, the distance weight was set as 'equal'. The Ensemble method was set as 'subspace', learner type was set as 'discriminant', the number of learners was set as 30 , and the subspace dimension was set as 13 .

To test the generalization ability of the three methods, $80 \%$ of the samples were randomly selected as the training dataset, and the remaining $20 \%$ were selected as the test dataset.

\section{Results}

ChlF induction curves under three different drought stress durations were measured. It cannot be seen that drought stress resulted in differences in the curves due to ChlF variations between samples within groups (Fig. 1).

Induction features were computed and subjected to the least significant difference (LSD) analysis to determine if they are statistically different for different durations of drought stress treatments (Fig. 2). $\mathrm{F}_{\mathrm{o}}, \mathrm{F}_{\mathrm{j}}, \mathrm{F}_{\mathrm{i}}, \mathrm{V}_{\mathrm{i}}, \mathrm{PI}_{\mathrm{ABS}}$, $\mathrm{ABS} / \mathrm{RC}, \mathrm{S}_{\mathrm{s}}, \mathrm{S}_{\mathrm{m}}, \mathrm{N}, \mathrm{M}_{0}, \mathrm{DI}_{0} / \mathrm{RC}$, and Area differed between the three drought levels. $\mathrm{V}_{\mathrm{j}}, \mathrm{F}_{\mathrm{m}} / \mathrm{F}_{\mathrm{o}}, \mathrm{F}_{\mathrm{v}} / \mathrm{F}_{\mathrm{o}}, \mathrm{F}_{\mathrm{v}} / \mathrm{F}_{\mathrm{m}}$, $\varphi \mathrm{E}_{0}, \varphi \mathrm{P}_{0}, \varphi \mathrm{D}_{0}, \psi_{0}$, and $\mathrm{HACH}$ Area do not show the

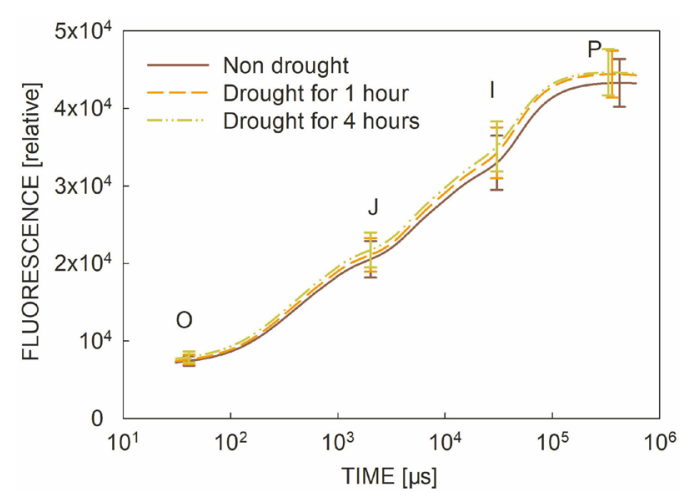

Fig. 1. Mean ChlF induction curves for three different drought levels (no drought, drought for $1 \mathrm{~h}$, and drought for $4 \mathrm{~h}$ ). The error bars are the standard deviations of $\mathrm{O}, \mathrm{J}, \mathrm{I}$, and $\mathrm{P}$ points of the rice samples under the same drought stress level. statistical difference between the non-drought (D0) and 1-h drought stress treatments (D1) while $\mathrm{F}_{\mathrm{m}}, \mathrm{F}_{\mathrm{v}}, \varphi \mathrm{Pav}$, and $\mathrm{ET}_{0} / \mathrm{RC}$ did not differ between the 1-h (D1) and 4-h (D4) drought stress treatments. Since the ChlF induction features varied, they are potentially useful for distinguishing drought levels in rice plants. It lays a base for the classification of different rice drought levels by using ChlF. However, the statistical difference does not mean that the classification accuracy for drought stress levels may be very high because of sample variations within groups.

Three classification techniques (SVM, KNN, and Ensemble) were applied to classify the plant samples into different drought levels with the commonly used maximum photochemical quantum yield of PSII in the dark $\left(\mathrm{F}_{\mathrm{v}} / \mathrm{F}_{\mathrm{m}}\right)$, the induction features, or the whole induction curve shown in Fig. 2. Table 1 shows the maximum classification accuracy (Max), the minimum classification accuracy (Min), and the average classification accuracy (Average) of running the test dataset 10 times by SVM, $\mathrm{KNN}$, and Ensemble, respectively. It can be seen that among the three classification methods, the average classification accuracy rate of the SVM method with the induction curve as input is the highest, which is $88.5 \%$. In addition, the induction curve as input performed better than just the induction features except for the KNN method.

To test the consistency of classification by the three methods (SVM, KNN, and Ensemble) for drought levels of rice, the Cohen's kappa coefficient was calculated to analyze the consistency between the actual stress levels and the predicted stress levels. Generally, the kappa coefficient is between 0 and 1 , which is divided into five intervals to represent the consistency between the actual category and the predicted category: $0.00-0.20$ indicates very low consistency, $0.21-0.40$ indicates fair consistency, 0.41-0.60 indicates moderate consistency, 0.61-0.80 indicates high consistency, and 0.81-1.00 indicates almost perfect consistency. The kappa coefficient values for the three classification methods are shown in Table 2. The kappa coefficient (0.82) of the SVM method with the induction curve as input is the highest. This result shows that SVM has great advantages in the qualitative analysis of rice drought levels.

For conciseness, only the results from SVM are further reported and discussed. The SVM classification results for the training dataset are shown in Fig. 3 and the classification results for the test dataset are listed in Table 4. Fig. 3 shows the confusion matrix of SVM classification of rice drought levels for the training dataset. The values in the confusion matrix represent the probability that the true drought stress levels are denoted by the vertical axis classified as the predicted drought stress levels denoted by the horizontal axis through the SVM classifier. The larger the diagonal value in the confusion matrix, the higher the classification accuracy. The total classification accuracy of drought stress levels is $47.0 \%$ (the mean of the truly positive results in Fig. $3 A$ ) only based on the maximum photochemical quantum yield of PSII $\left(\mathrm{F}_{\mathrm{v}} / \mathrm{F}_{\mathrm{m}}\right)$ in Fig. $3 A$. Fig. $3 B$ shows that the 

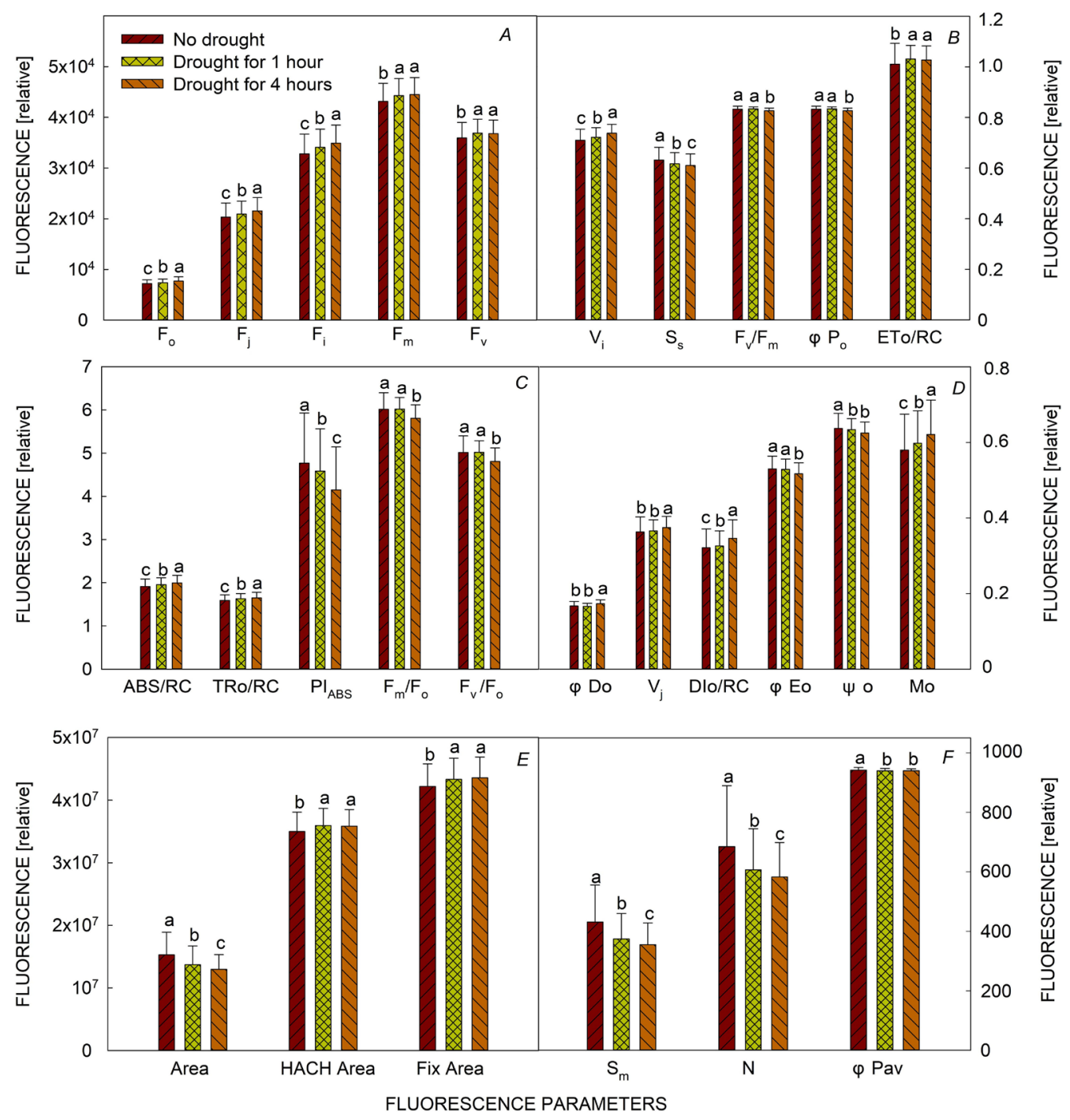

Fig. 2. One-way ANOVA of ChlF induction features under different drought levels (different lowercase letters indicate significant differences at $p<0.05) . \mathrm{F}_{\mathrm{o}}$ - minimal chlorophyll $a$ fluorescence intensity in the dark; $\mathrm{F}_{\mathrm{j}}$ - chlorophyll $a$ fluorescence intensity at the $\mathrm{J}$ step; $\mathrm{F}_{\mathrm{i}}$ - chlorophyll $a$ fluorescence intensity at the I step; $\mathrm{F}_{\mathrm{m}}$ - maximal chlorophyll $a$ fluorescence intensity; $\mathrm{F}_{\mathrm{v}}-$ variable fluorescence intensity in the dark; $V_{j}$ - relative variable fluorescence intensity at the J step; $V_{i}$ - relative variable fluorescence intensity at the I step; $\mathrm{F}_{\mathrm{m}} / \mathrm{F}_{\mathrm{o}}$ - electron transport through PSII; $\mathrm{F}_{\mathrm{v}} / \mathrm{F}_{\mathrm{o}}$ - quantum efficiency of PSII; $\mathrm{F}_{\mathrm{v}} / \mathrm{F}_{\mathrm{m}}$ - maximum photochemical quantum yield of PSII in the dark; $\mathrm{M}_{0}$ - approximated initial slope (in $\mathrm{ms}^{-1}$ ) of the fluorescence transient; Area - area between fluorescence curve and $\mathrm{F}_{\mathrm{m}}$ (background subtracted); Fix Area - area below the fluorescence curve between $F_{40 \mu s}$ and $F_{1 s}$ (background subtracted); $S_{m}-$ normalized area between ChlF induction curve and the line $\mathrm{F}=\mathrm{F}_{\mathrm{m}}$, (multiple turnover); $\mathrm{S}_{\mathrm{s}}$ - the smallest $\mathrm{S}_{\mathrm{m}}$ turnover (single turnover); $\mathrm{N}-$ reduction times of $\mathrm{Q}_{\mathrm{A}}$ from $\mathrm{F}_{\mathrm{o}}$ to $\mathrm{F}_{\mathrm{m}} ; \varphi \mathrm{P}_{0}$ - maximum quantum yield of PSII; $\psi_{0}$ - probability that a trapped exciton moves an electron further than $\mathrm{Q}_{\mathrm{A}}^{-} ; \varphi \mathrm{E}_{0}$ - quantum yield of electron transport; $\varphi \mathrm{D}_{0}$ - quantum yield of energy dissipation; $\varphi \mathrm{Pav}$ - average (from time 0 to $\mathrm{t}_{\mathrm{Fm}}$ ) quantum yield for primary photochemistry; $\mathrm{PI}_{\mathrm{ABS}}$ - performance index for energy conservation from exciton to the reduction of intersystem electron acceptors; $\mathrm{ABS} / \mathrm{RC}$ - absorption per reaction center; $\mathrm{TR}_{0} / \mathrm{RC}$ - trapped energy flux per reaction center; $\mathrm{ET}_{0} / \mathrm{RC}_{-}$electron transport per reaction center; $\mathrm{DI}_{0} / \mathrm{RC}$ - dissipation per reaction center $(a t \mathrm{t}=0)$.

total classification accuracy of drought stress levels can reach $73.3 \%$ (the total classification accuracy of the three drought levels based on the induction features, which is the mean of the true positive rate in Fig. $3 B$ ) and the correct classification rate for the 4-h drought stress treatment (D4) can reach $80.0 \%$ when only induction features were used as the SVM model input. However, the classification accuracy can reach $88.3 \%$ [the total classification accuracy of the three drought levels based on the induction curves, which is the mean of the truly positive rate in Fig. $3 C$ and the classification rate for the 4-h drought treatment (D4) can reach $91.0 \%$ when the data of the whole induction curve was used as the SVM model input (Fig. 3C)]. Most of the misclassified cases were classified into the neighbor groups. For example, samples of D4 have an $80 \%$ chance to be classified as drought 
Table 1. Comparison of drought stress level classification accuracy [\%] obtained from different methods (SVM, KNN, and Ensemble) and inputs for the test dataset. $\mathrm{F}_{\mathrm{v}} / \mathrm{F}_{\mathrm{m}}-$ maximum photochemical quantum yield of PSII.

\begin{tabular}{llllllllll}
\hline Methods & SVM & \multicolumn{3}{c}{ KNN } & \multicolumn{3}{c}{ Ensemble } \\
& Max & Min & Average & Max & Min & Average & Max & Min & Average \\
\hline $\mathrm{F}_{\mathrm{v}} / \mathrm{F}_{\mathrm{m}}[\%]$ & 46.24 & 43.59 & 45.45 & 45.13 & 38.46 & 41.95 & 44.62 & 40.51 & 41.91 \\
Induction features [\%] & 75.21 & 72.14 & 73.21 & 68.21 & 61.71 & 65.26 & 68.03 & 63.76 & 65.88 \\
Induction curve [\%] & 88.51 & 85.50 & 86.84 & 67.80 & 60.45 & 63.22 & 87.57 & 83.05 & 85.37 \\
\hline
\end{tabular}

Table 2. Comparison of the kappa coefficients obtained from different methods (SVM, KNN, and Ensemble) for the test dataset. $\mathrm{F}_{\mathrm{v}} / \mathrm{F}_{\mathrm{m}}$ - maximum photochemical quantum yield of PSII.

\begin{tabular}{llllllllll}
\hline Methods & SVM & \multicolumn{3}{c}{ KNN } & \multicolumn{3}{c}{ Ensemble } \\
& Max & Min & Average & Max & Min & Average & Max & Min & Average \\
\hline $\mathrm{F}_{\mathrm{v}} / \mathrm{F}_{\mathrm{m}}$ & 0.23 & 0.15 & 0.18 & 0.18 & 0.08 & 0.13 & 0.17 & 0.11 & 0.13 \\
Induction features & 0.63 & 0.58 & 0.60 & 0.52 & 0.43 & 0.48 & 0.52 & 0.46 & 0.49 \\
Induction curve & 0.83 & 0.78 & 0.82 & 0.52 & 0.41 & 0.45 & 0.81 & 0.75 & 0.78 \\
\hline
\end{tabular}

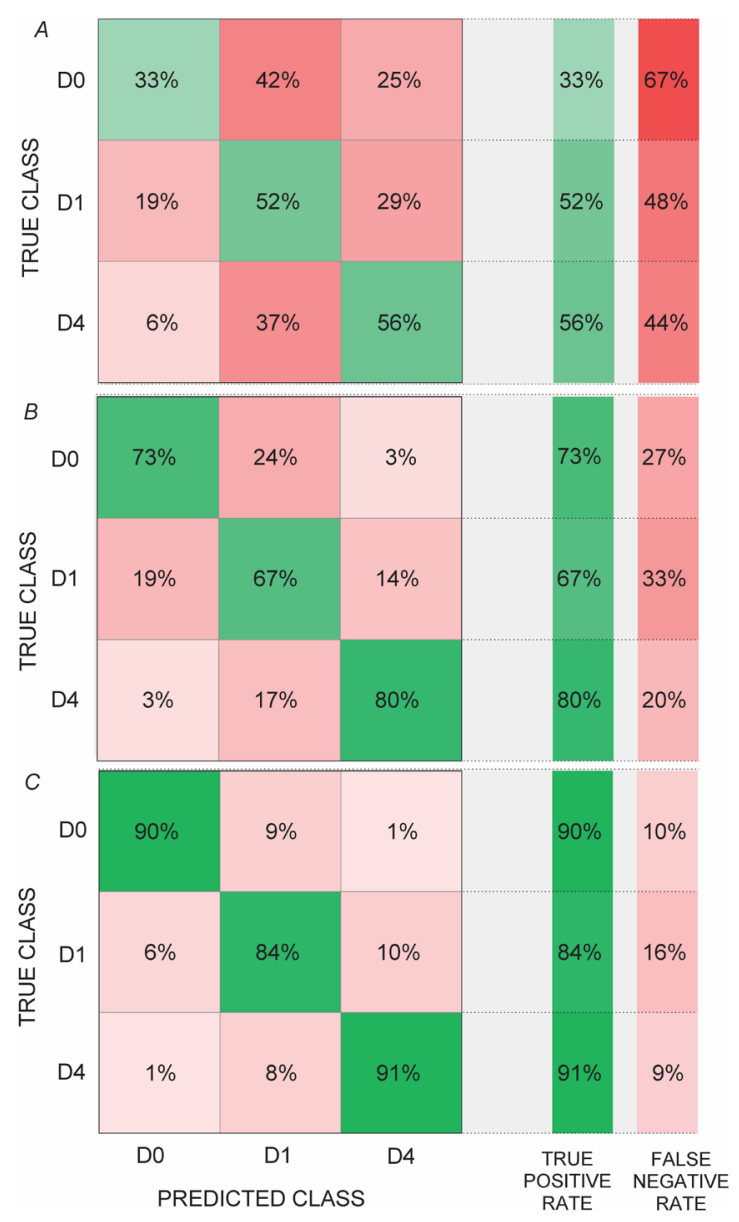

Fig. 3. Confusion matrix of Support Vector Machine (SVM) classification of rice drought levels for the training dataset. (A) Based on the maximum photochemical quantum yield of PSII $\left(\mathrm{F}_{\mathrm{v}} / \mathrm{F}_{\mathrm{m}}\right),(B)$ based on induction features, $(C)$ based on the induction curve. D0, D1, and D4 indicate no drought treatment, drought treatment for $1 \mathrm{~h}$, and drought treatment for $4 \mathrm{~h}$, respectively. treatment for 1-h (D1) but unlikely as non-drought rice (D0) in Fig. $3 C$.

Tables 3, 4, and 5 show the SVM classification results for the test dataset based on the $\mathrm{F}_{\mathrm{v}} / \mathrm{F}_{\mathrm{m}}$, induction features, and induction curve, respectively. Table 3 shows the classification accuracy for the total rice samples is $43.9 \%$ based on $F_{v} / F_{m}$ and the kappa coefficient value is 0.159 . The classification accuracy for the total rice samples is $72.7 \%$ based on induction features as shown in Table 4 and the classification accuracy for the total rice samples is $86.7 \%$ based on induction curves as shown in Table 5 . The total classification accuracy using the induction curves is $42.7 \%$ and $14.0 \%$ higher than that using $\mathrm{F}_{\mathrm{v}} / \mathrm{F}_{\mathrm{m}}$ and the induction features, respectively. In general, using the induction curve as input proved to be advantageous over using only $\mathrm{F}_{\mathrm{v}} / \mathrm{F}_{\mathrm{m}}$ or induction features as shown by the higher each or total correct classification percentages and a greater kappa coefficient value $(0.80$ vs. 0.60 or 0.16$)$. The correct classification rates using the induction curve for the non-drought treatment and 4-h drought treatment were relatively high in Table 5 , which should be related to the physiological changes of rice samples induced by drought stress.

\section{Discussion}

The difference of ChlF induction under different drought durations as shown in Fig. 1 and the statistical difference of some parameters under different drought durations as shown in Fig. 2 are consistent with findings in the literature. Under drought stress, different parts of electron transport of PSII are affected (Oukarroum et al. 2007, Zivcak et al. 2013, Bano et al. 2021). The changes of plant photosynthesis with external factors can be traced by measuring the ChlF induction and correlated with the drought stress (Saglam et al. 2014). The different steps and stages of ChlF induction can be linked to PSII, PSI, and the electron transfer efficiency between the two photosystems, components of photosynthetic electron transport 
Table 3. Classification results for test dataset based on the maximum photochemical quantum yield of PSII $\left(\mathrm{F}_{\mathrm{v}} / \mathrm{F}_{\mathrm{m}}\right)$. D0 - no drought stress treatment; D1 - drought stress treatment for $1 \mathrm{~h}$; D4 - drought stress treatment for $4 \mathrm{~h}$. Kappa coefficient $=0.16$.

\begin{tabular}{lcccl}
\hline \multicolumn{4}{c}{ Predicted samples } & \\
Actual samples & D0 & D1 & D4 & Total \\
\hline D0 & 63 & 81 & 51 & 195 \\
D1 & 48 & 87 & 60 & 195 \\
D4 & 15 & 73 & 107 & 195 \\
Total & 126 & 241 & 218 & 585 \\
Accuracy [\%] & 50.00 & 36.10 & 49.08 & 43.93 \\
\hline
\end{tabular}

Table 4. Classification results for test dataset based on induction features. D0 - no drought stress treatment; D1 - drought stress treatment for $1 \mathrm{~h}$; D4 - drought stress treatment for $4 \mathrm{~h}$. Kappa coefficient $=0.59$.

\begin{tabular}{lcccl}
\hline & \multicolumn{3}{l}{ Predicted samples } & \\
Actual samples & D0 & D1 & D4 & Total \\
\hline D0 & 142 & 45 & 8 & 195 \\
D1 & 37 & 128 & 30 & 195 \\
D4 & 5 & 35 & 155 & 195 \\
Total & 184 & 208 & 193 & 585 \\
Accuracy [\%] & 77.17 & 61.54 & 80.31 & 72.65 \\
\hline
\end{tabular}

Table 5. Classification results for testing dataset of rice under different drought levels using induction curve. D0 - no drought stress treatment; D1 - drought stress treatment for $1 \mathrm{~h}$; D4 drought stress treatment for $4 \mathrm{~h}$. Kappa coefficient $=0.80$.

\begin{tabular}{lcccl}
\hline \multicolumn{4}{c}{ Predicted samples } & \\
Actual samples & D0 & D1 & D4 & Total \\
\hline D0 & 169 & 24 & 2 & 195 \\
D1 & 13 & 169 & 13 & 195 \\
D4 & 3 & 21 & 171 & 195 \\
Total & 185 & 214 & 186 & 585 \\
Accuracy [\%] & 91.35 & 78.97 & 91.93 & 86.67 \\
\hline
\end{tabular}

(Stirbet and Govindjee 2011, Brestic et al. 2015). Previous studies found that ChlF has a strong correlation with rice drought stress. In our study, we expanded the research by proposing a machine learning method based on ChlF to classify drought levels.

Our results show that there are statistical differences in some induction features under different drought durations, which is consistent with the results in the literature (Kumar et al. 2016). The classification accuracy of using all the data on the OJIP induction curve as inputs is higher than the accuracy of only using $F_{v} / F_{m}$ or some ChlF feature parameters as inputs. In addition, the kappa coefficients in Table 5 also show that all the data on the OJIP induction curve as the model input lead to better classification performance for rice drought stress levels. This implies that drought stress level classification accuracy can be significantly improved by using the whole ChlF induction curve although there is no clear visible difference between ChlF signals for rice samples with different levels of drought stress treatment. This also implies that there is rich useful hidden biological information in the ChlF induction curve and the information can be used in future research for more application through machine learning techniques.

SVM is a supervised learning method. Its decision boundary is the maximum margin plane of the learning sample solution. In addition, the regularization term is added to the solution system to optimize the structural risk, so it has certain advantages in classification. Therefore, compared with the KNN and the Ensemble model, the SVM model has better capability to classify different levels of rice drought stress. And the kappa coefficients in Table 4 also show that the consistency of the classification results using SVM is relatively better. Machine learning methods are useful for the analysis of ChlF induction curves, especially in rice drought classification, which has been proved in this paper. This work demonstrated SVM had advantages in classifying rice drought stress levels with the ChlF data and the whole OJIP induction contains more plant physiological information.

Conclusion: In this work, the SVM technique was used for the classification of drought stress levels based on ChlF. The results show that the SVM model is a useful tool in rice drought stress level classification. In addition, the whole OJIP induction contains more information about the physiological state of plants, which was often omitted in literature when only the ChlF characteristic features are used in the analysis. In future work, more ChlF data under different environmental conditions should be considered to extend the developed method. It is expected that this ideal will be useful to improve agricultural production and agricultural genetic breeding under environmental stress.

\section{References}

Alam S., Kwon G.-R: Alzheimer disease classification using KPCA, LDA, and multi-kernel learning SVM. - Int. J. Imag. Syst. Tech. 27: 133-143, 2017.

Banks J.M.: Chlorophyll fluorescence as a tool to identify drought stress in Acer genotypes. - Environ. Exp. Bot. 155: 118-127, 2018.

Bano H., Athar H.U.R., Zafar Z.U. et al.: Linking changes in chlorophyll $a$ fluorescence with drought stress susceptibility in mung bean [Vigna radiata (L.) Wilczek]. - Physiol. Plantarum 172: 1244-1254, 2021.

Brestic M., Zivcak M., Kunderlikova K. et al.: Low PSI content limits the photoprotection of PSI and PSII in early growth stages of chlorophyll $b$-deficient wheat mutant lines. Photosynth. Res. 125: 151-166, 2015.

Cai S., Jiang G., Ye N. et al.: A key ABA catabolic gene, OsABA80x3, is involved in drought stress resistance in rice. PLoS ONE 10: e0116646, 2015.

Carrijo D.R., Akbar N., Reis A.F.B. et al.: Impacts of variable soil drying in alternate wetting and drying rice systems on yields, grain arsenic concentration and soil moisture dynamics. Field Crop. Res. 222: 101-110, 2018.

Faraloni C., Cutino I., Petruccelli R. et al.: Chlorophyll fluorescence technique as a rapid tool for in vitro screening of 
olive cultivars (Olea europaea L.) tolerant to drought stress. Environ. Exp. Bot. 73: 49-56, 2011.

Faseela P., Sinisha A.K., Brestič M., Puthur J.T.: Chlorophyll $a$ fluorescence parameters as indicators of a particular abiotic stress in rice. - Photosynthetica 58: 293-300, 2020.

Feng S., Fu L., Xia Q. et al.: Modelling and simulation of photosystem II chlorophyll fluorescence transition from darkadapted state to light-adapted state. - IET Syst. Biol. 12: 289293, 2018.

Fu L., Xia Q., Tan J. et al:: Modelling and simulation of chlorophyll fluorescence from PSII of a plant leaf as affected by both illumination light intensities and temperatures. - IET Syst. Biol. 13: 327-332, 2019.

Guerrero J.M., Pajares G., Montalvo M. et al.: Support vector machines for crop/weeds identification in maize fields. Expert Syst. Appl. 39: 11149-11155, 2012.

Guo Y., Tan J.: Recent advances in the application of chlorophyll $a$ fluorescence from photosystem II. - Photochem. Photobiol. 91: 1-14, 2015.

Haboudane D., Miller J.R., Pattey E. et al.: Hyperspectral vegetation indices and novel algorithms for predicting green LAI of crop canopies: Modeling and validation in the context of precision agriculture. - Remote Sens. Environ. 90: 337352,2004

Ignat T., Schmilovitch Z., Feföldi J. et al.: Nonlinear methods for estimation of maturity stage, total chlorophyll, and carotenoid content in intact bell peppers. - Biosyst. Eng. 114: 414-425, 2013.

Iturbe-Ormaetxe I., Escuredo P.R., Arrese-Igor C., Becana M.: Oxidative damage in pea plants exposed to water deficit or paraquat. - Plant Physiol. 116: 173-181, 1998.

Jiao W., Tian C., Chang Q. et al.: A new multi-sensor integrated index for drought monitoring. - Agr. Forest Meteorol. 268: $74-85,2019$.

Karimi Y., Prasher S.O., Patel R.M., Kim S.H.: Application of support vector machine technology for weed and nitrogen stress detection in corn. - Comput. Electron. Agr. 51: 99-109, 2006.

Kour V.P., Arora A.S.: Particle swarm optimization based support vector machine (P-SVM) for the segmentation and classification of plants. - IEEE Access 7: 29374-29385, 2019.

Kumar A., Lata C., Kumar P. et al.: Salinity and drought induced changes in gas exchange attributes and chlorophyll fluorescence characteristics of rice (Oryza sativa) varieties. Indian J. Agr. Sci. 86: 718-726, 2016.

Kumar S.V., Peters-Lidard C.D., Mocko D. et al.: Assimilation of remotely sensed soil moisture and snow depth retrievals for drought estimation. - J. Hydrometeorol. 15: 2446-2469, 2014.

Lazár D.: The polyphasic chlorophyll a fluorescence rise measured under high intensity of exciting light. - Funct. Plant Biol. 33: 9-30, 2006.

Maghsoudi K., Emam Y., Ashraf M.: Influence of foliar application of silicon on chlorophyll fluorescence, photosynthetic pigments, and growth in water-stressed wheat cultivars differing in drought tolerance. - Turk. J. Bot. 39: 625-634, 2015

Marcos-Garcia P., Lopez-Nicolas A., Pulido-Velazquez M.: Combined use of relative drought indices to analyze climate change impact on meteorological and hydrological droughts in a Mediterranean basin. - J. Hydrol. 554: 292-305, 2017.

Melandri G., AbdElgawad H., Riewe D. et al.: Biomarkers for grain yield stability in rice under drought stress. - J. Exp. Bot. 71: 669-683, 2020.

Meravi N., Prajapati S.K.: Temporal variation in chlorophyll fluorescence of different tree species. - Biol. Rhythm Res. 51: $331-337,2020$
Miyashita K., Tanakamaru S., Maitani T., Kimura K.: Recovery responses of photosynthesis, transpiration, and stomatal conductance in kidney bean following drought stress. Environ. Exp. Bot. 53: 205-214, 2005.

Mukherjee S., Mishra A., Trenberth K.E.: Climate change and drought: a perspective on drought indices. - Curr. Clim. Change Rep. 4: 145-163, 2018.

Oukarroum A., El Madidi S., Schansker G., Strasser R.J.: Probing the responses of barley cultivars (Hordeum vulgare L.) by chlorophyll $a$ fluorescence OLKJIP under drought stress and re-watering. - Environ. Exp. Bot. 60: 438-446, 2007.

Qiao X., Bao J., Zhang H. et al.: Underwater sea cucumber identification based on Principal Component Analysis and Support Vector Machine. - Measurement 133: 444-455, 2019.

Rosegrant M.W., Cline S.A.: Global food security: challenges and policies. - Science 302: 1917-1919, 2003.

Saglam A., Terzi R., Demiralay M.: Effects of polyethylene glycol induced drought stress on photosynthesis in two chickpea genotypes with different drought tolerance. - Acta Biol. Hung. 65: 178-188, 2014.

Sánchez N., González-Zamora Á., Martínez-Fernández J. et al.: Integrated remote sensing approach to global agricultural drought monitoring. - Agr. Forest Meteorol. 259: 141-153, 2018.

Sayed O.H.: Chlorophyll fluorescence as a tool in cereal crop research. - Photosynthetica 41: 321-330, 2003.

Stirbet A., Govindjee G.: On the relation between the Kautsky effect, chlorophyll $a$ fluorescence induction and photosystem II: basics and applications of the OJIP fluorescence transient. J. Photoch. Photobio. B 104: 236-257, 2011.

Timsorn K., Lorjaroenphon Y., Wongchoosuk C.: Identification of adulteration in uncooked Jasmine rice by a portable lowcost artificial olfactory system. - Measurement 108: 67-76, 2017.

Urban L., Aarrouf J., Bidel L.P.R.: Assessing the effects of water deficit on photosynthesis using parameters derived from measurements of leaf gas exchange and of chlorophyll $a$ fluorescence. - Front. Plant Sci. 8: 2068, 2017.

Wang F.Z., Wang Q.B., Kwon S.Y. et al.: Enhanced drought tolerance of transgenic rice plants expressing a pea manganese superoxide dismutase. - J. Plant Physiol. 162: 465-472, 2005.

Wang S., Tang H., Xia Q. et al.: Modeling and simulation of photosynthetic activities in $\mathrm{C}_{3}$ plants as affected by $\mathrm{CO}_{2}$. IET Syst. Biol. 13: 101-108, 2019.

Wang Y., Hu X., Hou Z. et al.: Discrimination of nitrogen fertilizer levels of tea plant (Camellia sinensis) based on hyperspectral imaging. - J. Sci. Food Agr. 98: 4659-4664, 2018.

Webber H., Ewert F., Olesen J.E. et al.: Diverging importance of drought stress for maize and winter wheat in Europe. - Nat. Commun. 9: 4249, 2018

West H., Quinn N., Horswell M.: Remote sensing for drought monitoring \& impact assessment: progress, past challenges and future opportunities. - Remote Sens. Environ. 232: $111291,2019$.

Woo N.S., Badger M.R., Pogson B.J.: A rapid, noninvasive procedure for quantitative assessment of drought survival using chlorophyll fluorescence. - Plant Methods 4: 27, 2008.

Xia Q., Tan J., Ji X. et al.: Modelling and simulation of chlorophyll fluorescence from photosystem II as affected by temperature. - IET Syst. Biol. 12: 304-310, 2018.

Xu Q., Ma X., Lv T. et al.: Effects of water stress on fluorescence parameters and photosynthetic characteristics of drip irrigation in rice. - Water 12: 289, 2020.

Yang X., Wang B., Chen L. et al.: The different influences of drought stress at the flowering stage on rice physiological traits, grain yield, and quality. - Sci. Rep.-UK 9: 3742, 2019. 
Zendonadi N.D.S., Piepho H.P., Condorelli G.E. et al.: Highthroughput field phenotyping reveals genetic variation in photosynthetic traits in durum wheat under drought. - Plant Cell Environ. 44: 2858-2878, 2021.
Zivcak M., Brestic M., Balatova Z. et al.: Photosynthetic electron transport and specific photoprotective responses in wheat leaves under drought stress. - Photosynth. Res. 117: 529-546, 2013.

Appendix. Chlorophyll $a$ fluorescence parameters.

\begin{tabular}{|c|c|}
\hline OJIP parameters & Definitions \\
\hline $\mathrm{F}_{\mathrm{o}}$ & Minimal chlorophyll $a$ fluorescence intensity in the dark \\
\hline $\mathrm{F}_{\mathrm{j}}$ & Chlorophyll $a$ fluorescence intensity at the J step \\
\hline $\mathrm{F}_{\mathrm{i}}$ & Chlorophyll $a$ fluorescence intensity at the I step \\
\hline $\mathrm{F}_{\mathrm{m}}$ & Maximal chlorophyll $a$ fluorescence intensity \\
\hline $\mathrm{F}_{\mathrm{v}}=\mathrm{F}_{\mathrm{m}}-\mathrm{F}_{\mathrm{o}}$ & Variable fluorescence intensity in the dark \\
\hline $\mathrm{V}_{\mathrm{j}}=\left(\mathrm{F}_{\mathrm{j}}-\mathrm{F}_{\mathrm{o}}\right) /\left(\mathrm{F}_{\mathrm{m}}-\mathrm{F}_{\mathrm{o}}\right)$ & Relative variable fluorescence intensity at the $\mathrm{J}$ step \\
\hline $\mathrm{V}_{\mathrm{i}}=\left(\mathrm{F}_{\mathrm{i}}-\mathrm{F}_{\mathrm{o}}\right) /\left(\mathrm{F}_{\mathrm{m}}-\mathrm{F}_{\mathrm{o}}\right)$ & Relative variable fluorescence intensity at the I step \\
\hline $\mathrm{F}_{\mathrm{m}} / \mathrm{F}_{\mathrm{o}}$ & Electron transport through photosystem II \\
\hline $\mathrm{F}_{\mathrm{v}} / \mathrm{F}_{\mathrm{o}}=\left(\mathrm{F}_{\mathrm{m}}-\mathrm{F}_{\mathrm{o}}\right) / \mathrm{F}_{\mathrm{o}}$ & Quantum efficiency of photosystem II \\
\hline $\mathrm{F}_{\mathrm{v}} / \mathrm{F}_{\mathrm{m}}=\left(\mathrm{F}_{\mathrm{m}}-\mathrm{F}_{\mathrm{o}}\right) / \mathrm{F}_{\mathrm{m}}$ & Maximum photochemical quantum yield of photosystem II in the dark \\
\hline $\mathrm{M}_{0}=4\left(\mathrm{~F}_{300}-\mathrm{F}_{\mathrm{o}}\right) /\left(\mathrm{F}_{\mathrm{m}}-\mathrm{F}_{\mathrm{o}}\right)$ & Approximated initial slope (in $\mathrm{ms}^{-1}$ ) of the fluorescence transient \\
\hline Area & Area between fluorescence curve and $\mathrm{F}_{\mathrm{m}}$ (background subtracted) \\
\hline Fix Area & Area below the fluorescence curve between $F_{40 \mu s}$ and $F_{1 s}$ \\
\hline $\mathrm{S}_{\mathrm{m}}=\operatorname{Area} /\left(\mathrm{F}_{\mathrm{m}}-\mathrm{F}_{\mathrm{o}}\right)$ & Normalized area between $\mathrm{ChlF}$ induction curve and the line $\mathrm{F}=\mathrm{F}_{\mathrm{m}}$ \\
\hline $\mathrm{S}_{\mathrm{s}}$ & The smallest $\mathrm{S}_{\mathrm{m}}$ turnover (single turnover) \\
\hline $\mathrm{N}=\mathrm{S}_{\mathrm{m}} \times \mathrm{M}_{0} \times\left(1 / \mathrm{V}_{\mathrm{j}}\right)$ & Reduction times of $\mathrm{Q}_{\mathrm{A}}$ from $\mathrm{F}_{\mathrm{o}}$ to $\mathrm{F}_{\mathrm{m}}$ \\
\hline$\varphi \mathrm{P}_{0}=1-\left(\mathrm{F}_{\mathrm{o}} / \mathrm{F}_{\mathrm{m}}\right)\left(\right.$ or $\left.\mathrm{F}_{\mathrm{v}} / \mathrm{F}_{\mathrm{m}}\right)$ & Maximum quantum yield of PSII \\
\hline$\psi_{0}=1-\mathrm{V}_{\mathrm{j}}$ & Probability that a trapped exciton moves an electron further than $\mathrm{Q}_{\mathrm{A}^{-}}^{-}$ \\
\hline$\varphi \mathrm{E}_{0}=\left(1-\mathrm{F}_{\mathrm{o}} / \mathrm{F}_{\mathrm{m}}\right) \times\left(1-\mathrm{V}_{\mathrm{j}}\right)$ & Quantum yield of electron transport \\
\hline$\varphi \mathrm{D}_{0}=1-\varphi \mathrm{P}_{0}=\mathrm{F}_{\mathrm{o}} / \mathrm{F}_{\mathrm{m}}$ & Quantum yield of energy dissipation \\
\hline$\varphi \mathrm{Pav}=\varphi \mathrm{P}_{0} \times\left(1-\mathrm{V}_{\mathrm{j}}\right)$ & Average (from time 0 to $t_{\mathrm{Fm}}$ ) quantum yield for primary photochemistry \\
\hline $\begin{array}{l}\mathrm{PI}_{\mathrm{ABS}}=\mathrm{RC} / \mathrm{ABS} \times\left[\varphi \mathrm{P}_{0} /\left(1-\varphi \mathrm{P}_{0}\right)\right] \times \\
\times\left[\psi_{0} /\left(1-\psi_{0}\right)\right]\end{array}$ & $\begin{array}{l}\text { Performance index for energy conservation from exciton to the reduction of intersystem } \\
\text { electron acceptors }\end{array}$ \\
\hline $\mathrm{ABS} / \mathrm{RC}=\left(\mathrm{M}_{0} / \mathrm{V}_{\mathrm{j}}\right)\left(1 / \varphi \mathrm{P}_{0}\right)$ & Absorption per reaction center \\
\hline $\mathrm{TR}_{0} / \mathrm{RC}=\mathrm{M}_{0} \times\left(1 / \mathrm{V}_{\mathrm{j}}\right)$ & Trapped energy flux per reaction center \\
\hline $\mathrm{ET}_{0} / \mathrm{RC}=\left(\mathrm{M}_{0} / \mathrm{V}_{\mathrm{j}}\right)\left(1-\mathrm{V}_{\mathrm{j}}\right)$ & Electron transport per reaction center \\
\hline $\mathrm{DI}_{0} / \mathrm{RC}=\mathrm{ABS} / \mathrm{RC}-\mathrm{TR}_{0} / \mathrm{RC}$ & Dissipation per reaction center $($ at $t=0$ ) \\
\hline
\end{tabular}

(C) The authors. This is an open access article distributed under the terms of the Creative Commons BY-NC-ND Licence. 